\title{
Antisense Expression of the Arabidopsis thaliana AtPGIP1 Gene Reduces Polygalacturonase-Inhibiting Protein Accumulation and Enhances Susceptibility to Botrytis cinerea
}

\author{
Simone Ferrari, Roberta Galletti, Donatella Vairo, Felice Cervone, and Giulia De Lorenzo \\ Università degli Studi “La Sapienza”, Dipartimento di Biologia Vegetale, Piazzale Aldo Moro 5, 00185 Roma, Italy \\ Submitted 2 November 2005. Accepted 31 March 2006.
}

Polygalacturonases (PGs) hydrolyze the homogalacturonan of plant cell-wall pectin and are important virulence factors of several phytopathogenic fungi. In response to abiotic and biotic stress, plants accumulate $P G$-inhibiting proteins (PGIPs) that reduce the activity of fungal PGs. In Arabidopsis thaliana, PGIPs with comparable activity against BcPG1, an important pathogenicity factor of the necrotrophic fungus Botrytis cinerea, are encoded by two genes, AtPGIP1 and AtPGIP2. Both genes are induced by fungal infection through different signaling pathways. We show here that transgenic Arabidopsis plants expressing an antisense AtPGIP1 gene have reduced AtPGIP1 inhibitory activity and are more susceptible to $B$. cinerea infection. These results indicate that PGIP contributes to basal resistance to this pathogen and strongly support the vision that this protein plays a role in Arabidopsis innate immunity.

Additional keyword: pectic enzymes.

The cell wall is the first barrier that plant cells oppose to the attack of pathogens. In order to breach this barrier and gain access to the plant cell, most phytopathogenic microorganisms produce a battery of enzymes capable of degrading cell-wall polymers. Among them, pectic enzymes (polygalacturonases, pectate lyases, and pectin lyases) are responsible for the early degradation of pectin of the middle lamella and primary cell wall (De Lorenzo et al. 1997). Polygalacturonases (PGs, EC 3.2.1.15), which cleave the $\alpha-(1 \rightarrow 4)$ linkages between D-galacturonic acid residues in nonmethylated homogalacturonan, a major component of pectin, have been extensively studied. PG activity leads to the maceration of host tissue and the release of nutrients necessary for pathogen growth. Targeted genedisruption experiments indicate that PGs are important virulence factors in Botrytis cinerea, whose BcPG1 and BcPG2 are required for full virulence (Kars et al. 2005; ten Have et al. 1998), as well as in other fungi (Isshiki et al. 2001; Oeser et al. 2002; Shieh et al. 1997) and some bacteria (Huang and Allen 2000).

S. Ferrari and R. Galletti contributed equally to the paper.

Corresponding author: G. De Lorenzo;

E-mail: giulia.delorenzo@uniroma1.it

Current address for S. Ferrari: Università degli Studi di Padova, Dipartimento Territorio e Sistemi Agro-forestali, Viale dell'Università 16, 35020 Legnaro (PD), Italy.
Specific PG-inhibiting proteins (PGIPs) are present in the cell walls of all plants so far examined (De Lorenzo et al. 2001). PGIPs can specifically inhibit fungal and insect PGs (D'Ovidio et al. 2004) and are usually ineffective against enzymes of either bacterial or plant origin (Cervone et al. 1990). The interaction between fungal PGs and plant PGIPs might also favor the production of elicitors at the site of infection. In vitro experiments have demonstrated that addition of PGIP to fungal PGs in the presence of polygalacturonic acid results in the accumulation in the reaction mixture of oligogalacturonides with elicitor activity (Cervone et al. 1989).

In Arabidopsis thaliana, two genes, AtPGIP1 and AtPGIP2, encode inhibitors with similar efficiency against BcPG1 of $B$. cinerea (Ferrari et al. 2003b; Manfredini et al. 2005) but with different activity against a PG from Colletotrichum acutatum (Ferrari et al. 2003b). Overexpression of either AtPGIP1 or AtPGIP2 in Arabidopsis reduces disease symptoms caused by B. cinerea (Ferrari et al. 2003b). Since both AtPGIPl and AtPGIP2 are induced by $B$. cinerea with similar kinetics (Ferrari et al. 2003b), it is possible that expression of both genes is required to achieve the basal level of resistance observed in untransformed Arabidopsis plants. However, the observation that induction of AtPGIPl is mediated by oligogalacturonides whereas AtPGIP2 expression is mediated by methyl jasmonate (Ferrari et al. 2003b) also suggests that the two genes were maintained during evolution because of their different regulation. Overexpression experiments do not allow us to determine whether induction of specific PGIPs in wild-type plants is actually necessary to restrict fungal infection. In this paper, we show that expression of AtPGIPl in antisense orientation results in dramatically reduced levels of the inducible inhibitory activity in Arabidopsis plants. Antisense plants also have enhanced susceptibility to $B$. cinerea infection, indicating that accumulation of PGIP is required for full resistance to this pathogen.

\section{RESULTS}

Selection of antisense AtPGIP1 plants.

A. thaliana Col-0 plants expressing an expressed sequence tag (EST) clone corresponding to AtPGIP1 in antisense orientation under the control of the Cauliflower mosaic virus $35 \mathrm{~S}$ promoter were generated by Agrobacterium-mediated transformation. About 50 primary transformants (T1) were selected for kanamycin resistance and were allowed to self and produce segregating progeny (T2). RNA blot analysis of kanamycinresistant seedlings from 10 randomly chosen $\mathrm{T} 2$ lines allowed 
us to identify two transgenic lines, AS3 and AS4, segregating 3:1 for kanamycin resistance and accumulating high levels of antisense transcripts. No antisense transcripts were detected in untransformed plants or in plants transformed with a control plasmid (line pBI) (Fig. 1A). All transgenic lines showed normal morphology and fertility (data not shown).

We have previously shown that AtPGIP1 activity in untreated Arabidopsis seedlings is very low, but that it significantly increases upon cold treatment whereas low temperature does not induce AtPGIP2 expression (Ferrari et al. 2003b). Therefore, in order to determine the effect of the expression of the antisense transcript on AtPGIP1 accumulation, wild-type seedlings and kanamycin-resistant $\mathrm{T} 2$ antisense seedlings were incubated for $72 \mathrm{~h}$ at $+4^{\circ} \mathrm{C}$. Total protein extracts were analyzed for the presence of inhibitory activity against $B$. cinerea $\mathrm{PG}$, which is strongly inhibited by AtPGIP1 (Ferrari et al. 2003b). No inhibitory activity was observed in untreated seedlings of control and transgenic lines. A significant increase of activity was ob-
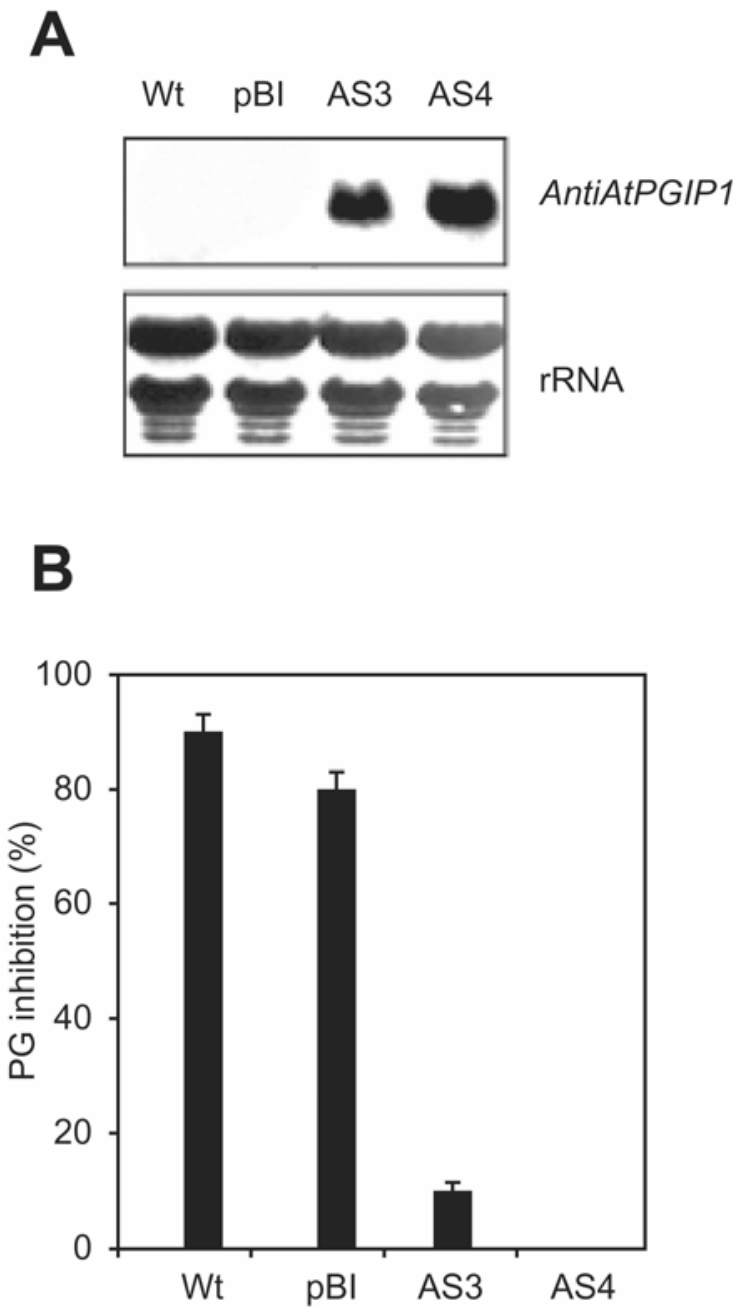

Fig. 1. Characterization of antisense AtPGIP1 plants. A, RNA blot of untreated 10-day-old seedlings hybridized with a single-strand probe recognizing antisense AtPGIPl transcripts (AntiAtPGIP1). To verify equal loading, ribosomal RNA was visualized by methylene blue staining ( $r R N A)$. B, Inhibition of polygalacturonase (PG) activity of Botrytis cinerea (1 agarose diffusion unit) was measured in the presence of $3 \mu \mathrm{g}$ of plant protein extracts from 10-day-old seedlings incubated for $72 \mathrm{~h}$ at $+4{ }^{\circ} \mathrm{C}$ and were compared with the activity of an identical amount of PG alone (100\%). Bars indicate average \pm standard error of three replicates. Wt $=$ untransformed plants, $\mathrm{pBI}=$ control plants transformed with the $\mathrm{pBI} 121$ vector, and AS3 and AS4 = plants from two independent lines transformed with the AtPGIP1 antisense construct. served after $72 \mathrm{~h}$ of cold treatment in wild-type plants as well as in the control line pBI, whereas the same amount of total protein extracts from the cold-treated antisense line AS4 failed to inhibit $B$. cinerea PG (Fig. 1B). A strong reduction in inhibitory activity was also detected in cold-treated AS3 plants (Fig. 1B). Homozygous T3 AS3, AS4, and pBI lines were obtained and used for further characterization.

To determine whether expression of AtPGIP2 is affected in antisense plants, control and antisense liquid-grown seedlings were treated with methyl jasmonate (MeJA), which induces accumulation of AtPGIP2 but not of AtPGIP1 (Ferrari et al. 2003 b). A very low inhibitory activity against $B$. cinerea $P G$ could be detected in all untreated seedlings; after MeJA treatment this activity increased to a similar extent in all lines with the exception of AS3, which showed a slight reduction of inducible activity (Fig. 2A). To ensure that the activity observed in MeJA-treated plants was due to AtPGIP2, we analyzed the ability of the same protein extracts to inhibit a PG from $C$. acutatum. This enzyme allows discrimination between the two Arabidopsis inhibitors, since its activity is inhibited by AtPGIP2 about 10-fold less than by AtPGIP1 (Ferrari et al. 2003b). Consistent with an expected induction of AtPGIP2, MeJA-induced inhibitory activity against $C$. acutatum PG was much lower than that against $B$. cinerea $P G$ in all tested lines (Fig. 2B). We also analyzed the effect of the expression of the AtPGIP1 antisense transcript on AtPGIP transcript levels by quantitative reverse transcript-polymerase chain reaction (RTPCR). A comparable increase in AtPGIP2 transcript levels in response to MeJA was observed in both untransformed and antisense plants (Fig. 2C); slight differences were due to biological variation. AtPGIPl expression increased about fivefold upon MeJA treatment in untransformed plants. However, AtPGIP1 mRNA levels were much lower than AtPGIP2 levels (Fig. 2C); this explains our previous failure to detect AtPGIPl expression in MeJA-treated plants by RNA blot analysis and inhibitor activity assays (Ferrari et al. 2003b; this work). Induction of AtPGIP1 by MeJA in AS3 plants was almost two times lower than in untransformed plants (Fig. 2C).

Taken together, these results indicate that induction of AtPGIP1 expression upon abiotic stress is strongly reduced in both antisense lines, whereas expression of AtPGIP2 is not significantly affected.

\section{Antisense AtPGIP1 plants are more susceptible to $B$. cinerea.}

In order to determine whether the observed reduction of PGIP activity in antisense plants impaired pathogen resistance, rosette leaves from untransformed and antisense plants were inoculated with $B$. cinerea and the development of disease symptoms was monitored for $60 \mathrm{~h}$ after infection. As a control line with known enhanced susceptibility to $B$. cinerea, we also inoculated ein2 plants, which are defective in ethylene signaling (Alonso et al. 1999). We have previously shown that ein2 leaves develop greater lesions upon $B$. cinerea infection (Ferrari et al. 2003a). The number of inoculation sites resulting in expanding, water-soaked lesions dramatically increased in both antisense lines as well as in ein2 plants (Fig. 3A). Furthermore, the average area of the lesions in AS3, AS4, and ein2 leaves was significantly greater than in untransformed plants (Fig. 3B). Both percentage of developing lesions and average lesion area were comparable in antisense and ein2 plants (Fig. 3A and B). No significant difference in susceptibility was observed between untransformed and $\mathrm{pBI}$ plants (data not shown).

The accumulation of AtPGIP1 and AtPGIP2 transcripts in antisense plants inoculated with $B$. cinerea was determined by quantitative RT-PCR experiments performed on healthy and infected leaves. Inoculation of untransformed plants with $B$. 
cinerea caused a 100-fold increase of AtPGIP1 expression (Fig. 4) and an about 10-fold increase of AtPGIP2 expression, which reached levels comparable to those of AtPGIPI (Fig. 4). In contrast, accumulation of AtPGIPI mRNA upon infection was reduced strongly and to a similar extent in AS3 and AS4 plants (Fig. 4). Unexpectedly, AtPGIP2 mRNA levels were also reduced in AS3 and, to a lesser extent, AS4 infected leaves (Fig. 4).

\section{DISCUSSION}

Plants have evolved a battery of defensive proteins in order to fend off pathogen attacks. A large amount of evidence supports a role for several of them in protection against specific microbial pathogens. In many cases, this evidence was obtained generating transgenic plants overexpressing specific proteins. For instance, constitutive expression of a radish defensin in tobacco confers resistance to the fungus Alternaria longipes, indicating that this protein has antifungal properties in vivo (Terras et al. 1995). Similarly, overexpression of an endogenous thionin enhances resistance of Arabidopsis against Fusarium oxysporum (Epple et al. 1997). The approach of overexpression has been used for a variety of proteins and has important biotechnological implications. However, an increase of pathogen resistance upon overexpression of a protein does not give any conclusive information about its role in the parental, untransformed plant. In other terms, even if a protein improves resistance when constitutively expressed at very high levels, it may contribute only marginally to the basal level of resistance observed in untransformed plants, because its induction may be too slow or too weak to exert a significant impact on pathogen growth.

Several defense-related proteins are encoded by multigenic families that may have partial or complete functional redundancy. As a consequence, the real contribution of specific family members to basal resistance may be difficult to assess. In a previous work, we have shown that Arabidopsis PGIPs are encoded by two closely related genes (AtPGIP1 and AtPGIP2) with similar inhibitory activity against the PGs produced in culture by $B$. cinerea (Ferrari et al. 2003b) as well as BcPG1 heterologously expressed in Pichia pastoris (Manfredini et al. 2005). Overexpression of either AtPGIP1 or AtPGIP2 leads to enhanced resistance against this pathogen (Ferrari et al. $2003 \mathrm{~b}$ ), supporting the hypothesis of at least a partial overlap of functions.
To determine whether expression of PGIPs during infection is required for basal resistance to fungal infection, we have expressed AtPGIPI in antisense orientation in Arabidopsis. We isolated two independent antisense plants that have greatly re-
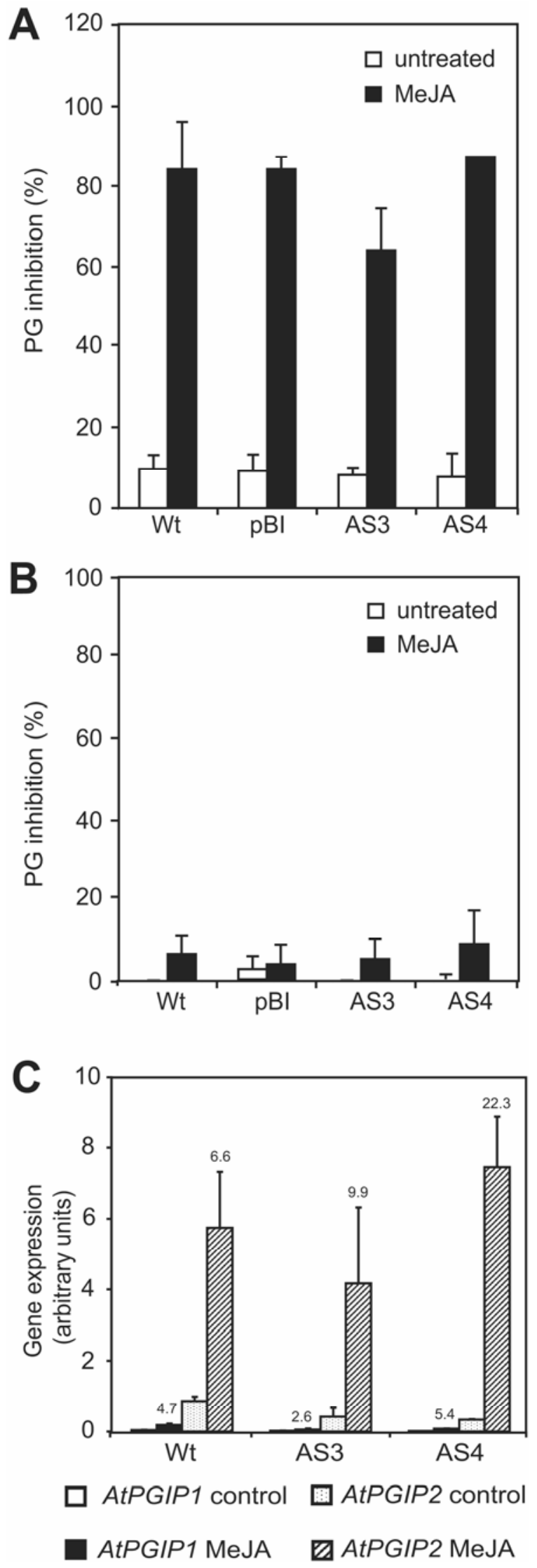

Fig. 2. Polygalacturonase-inhibiting protein (PGIP) expression in antisense lines after methyl jasmonate (MeJA) treatment. A and B, Crude protein extracts were prepared from 10-day-old seedlings grown for $72 \mathrm{~h}$ in the presence (black bars) or the absence (white bars) of $43 \mu \mathrm{M}$ MeJA and were tested for inhibitory activity. A, Botrytis cinerea or B, Colletotrichum acutatum PG activity (corresponding to 0.7 and 1 agar diffusion unit, respectively) was measured in the presence of $3 \mu \mathrm{g}$ of plant protein extracts and was compared with the activity of an identical amount of PG alone. Bars indicate average \pm standard error of three replicates. Wt $=$ untransformed plants, $\mathrm{pBI}=$ control plants transformed with the pBI121 vector, AS3 and AS4 = plants transformed with the AtPGIP1 antisense construct. C, Expression of AtPGIP1 (white and black bars) and AtPGIP2 (dotted and striped bars) in plants treated with MeJA. Absolute levels of PGIP transcripts were determined in control (white and dotted bars) and MeJA-treated seedlings (black and striped bars) by quantitative reverse transcript-polymerase chain reaction, and relative expression in each sample was obtained by normalization with the expression of the $U B Q 5$ gene. Bars indicate average expression levels for two replicates, expressed in arbitrary units \pm standard deviation. The average fold-change expression of AtPGIP1 or AtPGIP2 in MeJAtreated seedlings of each genotype compared with the basal levels in untreated plants of the same genotype is indicated above the corresponding bar. $\mathrm{Wt}=$ untransformed plants, AS3 and AS4 = antisense plants. 
duced induction of AtPGIP1 expression upon abiotic and biotic stresses and found that both lines have enhanced susceptibility towards B. cinerea. Antisense technology has previously been employed to determine the importance of plant defense-related proteins. In the case of tobacco class I endo-1,3-beta-glucanases antisense expression, endogenous levels of the protein was effectively reduced with no significant impact on pathogen resistance, possibly because other compensatory enzymatic activities are induced during infection (Beffa et al. 1993). Similarly, po-

A

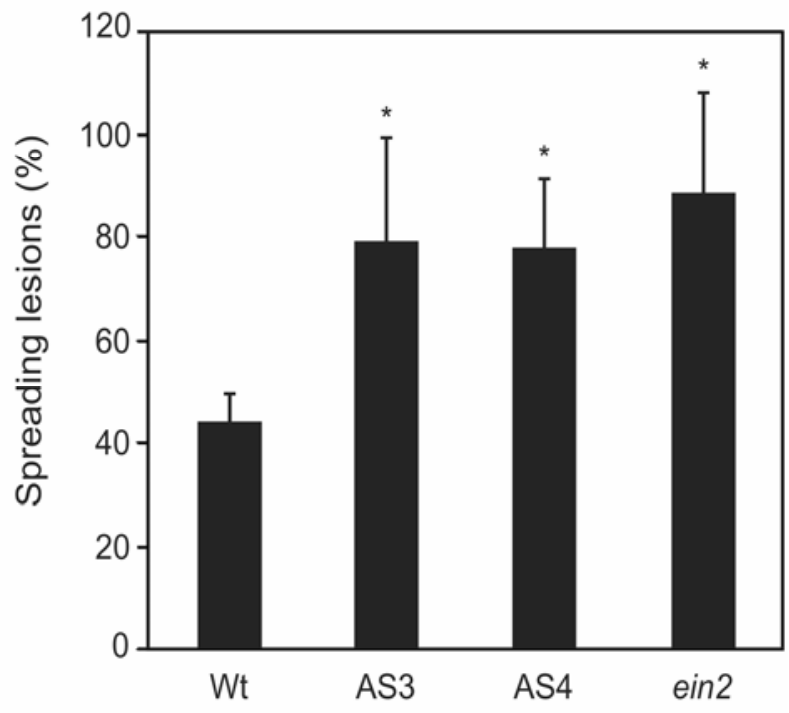

B

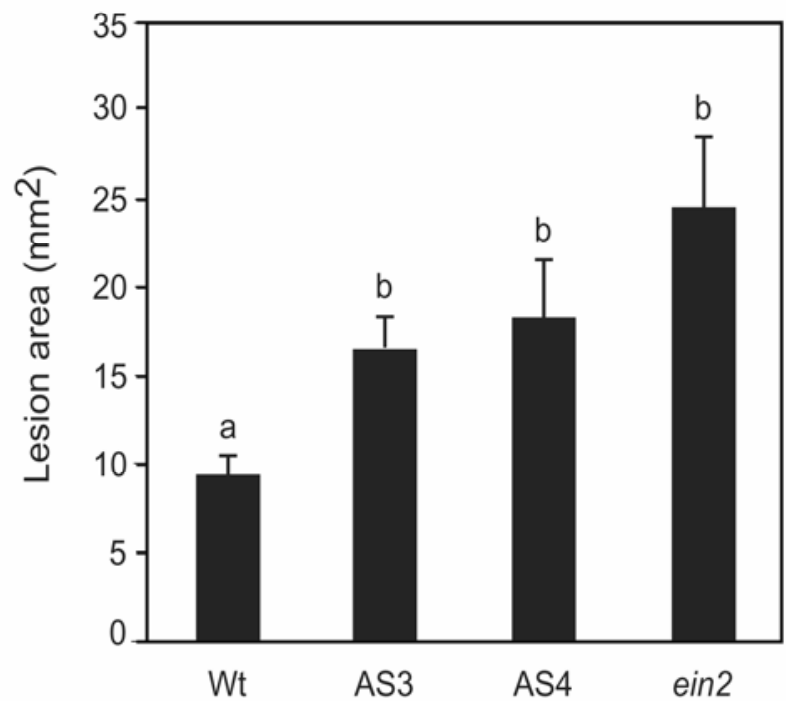

Fig. 3. Susceptibility of antisense lines to Botrytis cinerea infection. A, Rosette leaves of 4-week-old untransformed (Wt), antisense (AS3, AS4), and ein2-1 plants were inoculated with $B$. cinerea, and the percentage of infections resulting in water-soaked, spreading lesions was determined after $60 \mathrm{~h}$. Bars represent average percentages from three independent experiments \pm standard deviation. Asterisks indicate data sets significantly different from the wild type according to the Student's $t$-test $(P<0.05)$. B, Rosette leaves of 4-week-old untransformed (Wt), antisense (AS3, AS4), and ein2-1 plants were inoculated with $B$. cinerea, and the area of spreading lesions was determined after $60 \mathrm{~h}$. Data represent average area of $>10$ lesions \pm standard error Different letters indicate data sets significantly different, according to oneway analysis of variance followed by Newman-Keuls range test. This experiment was repeated three times with similar results. tato plants expressing an osmotin-like protein in antisense orientation showed decreased accumulation of the corresponding mRNA and protein when challenged by fungal infection but did not exhibit any alteration in disease susceptibility (Zhu et al. 1996). This does not appear to be the case of PGIP and we have demonstrated that its expression not only is sufficient to confer enhanced resistance to $B$. cinerea in transgenic Arabidopsis plants (Ferrari et al. 2003b) but is also necessary in wild-type plants for basal resistance to this fungus (this work). PGIP levels, therefore, contribute to determine the degree of symptom development observed in Arabidopsis plants upon B. cinerea infection. In support of our findings, a recent study shows that a quantitative trait locus associated with $B$. cinerea susceptibility in Arabidopsis maps in the chromosome region harboring AtPGIP1 and AtPGIP2 (Denby et al. 2004), suggesting that PGIPs play a major role in horizontal resistance to this pathogen. Our results also confirm that PGIPs are not involved in physiological processes. Like antisense AtPGIP1 plants, plants overexpressing AtPGIP1 or AtPGIP2 do not have obvious developmental defects (Ferrari et al. 2003b). In contrast, it has been reported that expression of a rice PGIP gene (OsFORI) in antisense orientation causes an increase in the number of floral organs (Jang et al. 2003).

Our data complement our previous observations on the defensive potential of AtPGIP1 and AtPGIP2 against $B$. cinerea. Although we did not observe a significant reduction in AtPGIP2 activity or AtPGIP2 mRNA levels in the antisense plants treated with MeJA, these plants showed an unexpected reduction of AtPGIP2 mRNA levels upon fungal infection, which can be explained by the similarity between the two genes (78 and $76 \%$ identity at the nucleotide and amino acid level, respectively) (Ferrari et al. 2003b). The negligible effect of the antisense expression on AtPGIP2 in MeJA-treated plants may be a consequence of the very low levels of AtPGIPl transcripts accumulated upon this treatment, which may not be sufficient to trigger the suppression of the closely related

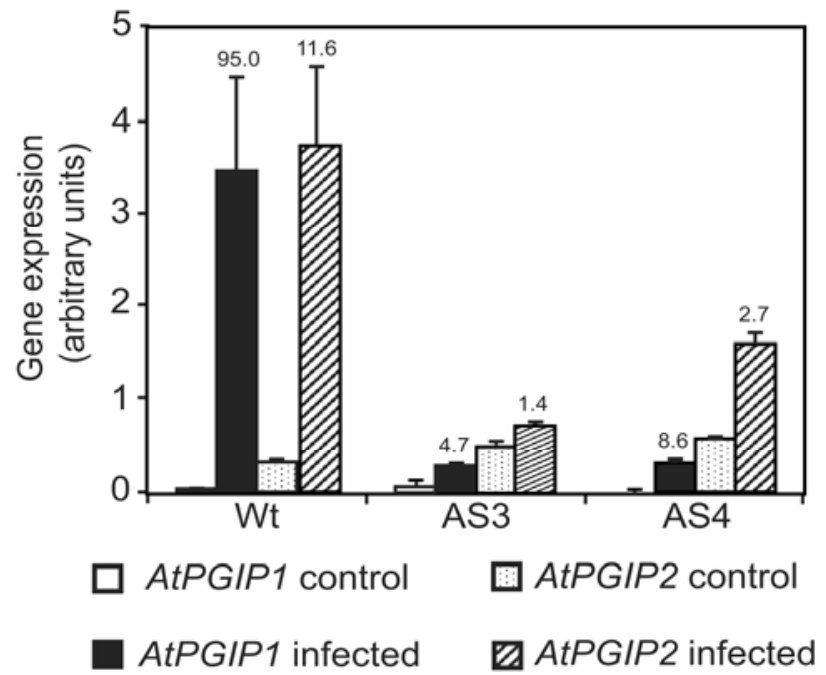

Fig. 4. Expression of AtPGIP genes in infected plants. Absolute levels of AtPGIP1 (white and black bars) and AtPGIP2 (dotted and striped bars) transcripts were determined in uninfected rosette leaves (white and dotted bars) and in leaves inoculated with Botrytis cinerea at 60 hpi (black and striped bars) by quantitative reverse transcript-polymerase chain reaction, and relative expression in each sample was obtained by normalization with the expression of the $U B Q 5$ gene. Bars indicate average expression levels for three replicates, expressed in arbitrary units \pm standard deviation. The average fold-change expression of AtPGIP1 or AtPGIP2 in untreated leaves of each genotype, as compared with the basal level in the inoculated leaves of the same genotype, is indicated above the corresponding bar. Wt $=$ untransformed plants, AS3 and AS4 = antisense plants. 
AtPGIP2. Furthermore, biotic and abiotic stresses can affect RNA interference (Madlung and Comai 2004) and increase gene silencing (Meza et al. 2001). Silencing of AtPGIP2 upon infection or in the infected rosette leaves may be higher than in the MeJA-treated seedlings. However, since silencing of AtPGIP2 was only partial, all this is largely a matter of speculation, and conclusions about the distinct roles of AtPGIP1 and AtPGIP2 in defense against $B$. cinerea cannot be drawn. Both are expressed at the site of infection and encode proteins with similar activity; thus, both may contribute to restrict $B$. cinerea growth (Ferrari et al. 2003b). Regulation of the two inhibitors through two distinct pathways may confer a selective advantage; if a pathogen suppresses the activation of one pathway, at least one gene is still expressed.

In conclusion, in this work, we have shown that antisense expression of the AtPGIP1 gene in Arabidopsis effectively reduces accumulation of the corresponding inhibitor activity in response to stress stimuli. Interestingly, AtPGIP1 antisense expression also partially reduces $A t P G I P 2$ expression in infected leaves. The final outcome of the reduced expression of the two PGIP genes is an enhanced susceptibility to the fungal pathogen $B$. cinerea. Our results therefore highlight the importance of PGIP as a defense protein against fungal infection and strongly support its role in Arabidopsis innate immunity (Federici et al. 2006).

\section{MATERIALS AND METHODS}

Plant growth and treatments.

Arabidopsis thaliana ecotype Columbia-0 (Col-0) plants were grown on soil in a growth chamber at $22^{\circ} \mathrm{C}$, relative humidity of $70 \%$, and a 12-h-light and 12-h-dark photoperiod $\left(120 \mu \mathrm{E} \mathrm{m}^{-2} \mathrm{~s}^{-1}\right)$, unless indicated otherwise. Cold treatments were conducted on 10-day-old seedlings grown at the temperature and photoperiod indicated above on agar plates containing Murashige and Skoog (MS) medium with Gamborg's B5 vitamins (Sigma, St. Louis) and 1\% sucrose. Plates were wrapped in aluminum foil and placed at $+4^{\circ} \mathrm{C}$ for $72 \mathrm{~h}$ before harvesting. For MeJA treatments, seedlings were grown in the same conditions in liquid MS medium with Gamborg's B5 vitamins and $0.5 \%$ sucrose. After 10 days, MeJA (Fluka, Buchs, Switzerland) was added to the medium at a final concentration of $43 \mu \mathrm{M}$, and seedlings were harvested after $72 \mathrm{~h}$.

\section{Fungal cultures and infections.}

Botrytis cinerea B05.10 and Colletotrichum acutatum isolate SHK788 were gifts from P. Tudzynski (Institut fur Botanik Westfalische Wilhelms-Universitat, Munster, Germany) and D. Berger (University of Pretoria, South Africa), respectively. The fungi were cultured as described by Ferrari and associates (2003b). In that study, C. acutatum was indicated as C. gleosporioides. B. cinerea infections were conducted as previously described (Ferrari et al. 2003b). For each experiment, at least five leaves for each genotype were inoculated with two $5-\mu 1$ droplets containing $5 \times 10^{5}$ spores per milliliter in potato dextrose broth (Fluka) at $24 \mathrm{~g} \mathrm{ml}^{-1}$. The diameter of each lesion was measured after $60 \mathrm{~h}$ with a ruler, and lesion area was calculated as follows: area $=\left[3.14 \times(\text { diameter } / 2)^{2}\right]$. Data were subjected to one-way analysis of variance followed by NewmanKeuls range test.

\section{Generation of transgenic lines.}

The insert of the A. thaliana Col-0 EST clone 179F6T7 (GenBank accession number H36821) was completely sequenced and corresponded to a full-length AtPGIP1 cDNA, except for a 200-bp deletion in the first exon (from position +158 to +358 , relative to the start codon), probably due to a cloning error during the generation of the EST library. This deletion, however, does not result in a frameshift, and the clone was therefore used to generate the transgenic plants. The cDNA insert of the EST clone was excised with BamHI and SmaI and was inserted in antisense orientation downstream of the Cauliflower mosaic virus $35 \mathrm{~S}$ promoter in the binary vector pBI121 (Clontech, Palo Alto, CA, U.S.A.), recovered after linearization with SstI, filling in with Klenow DNA polymerase, and excision of the uidA gene with BamHI. Adult plants (T0) were transformed with the antisense construct or with pBI121, using Agrobacterium tumefaciens GV3101 by floral dipping (Clough and Bent 1998). Transformants (T1) were selected on agar plates supplemented with MS medium, $1 \%$ sucrose, and $50 \mu \mathrm{g}$ of kanamycin per milliliter, were transferred to soil and allowed to self-fertilize, and the progeny of individual transformants was propagated (T2 generation).

\section{Protein extraction and determination of polygalacturonase activity.}

Fungal growth and PG preparation were previously described (Ferrari et al. 2003b). Total plant proteins were extracted by homogenization in $1 \mathrm{M} \mathrm{NaCl}, 20 \mathrm{mM}$ sodium acetate, $\mathrm{pH} 4.7$ (2 $\mathrm{ml}$ per gram of fresh weight). Homogenates were incubated at $+4^{\circ} \mathrm{C}$ for $1 \mathrm{~h}$ and were centrifuged for $20 \mathrm{~min}$ at $8,000 \times g$. Total protein concentration in the supernatant was determined with the Bradford method (Bradford 1976) (Biorad, Munich, Germany). PG activity was measured using a modified agarose diffusion assay (Taylor and Secor 1988). A solution containing PG or culture filtrates was added to $0.5-\mathrm{cm}$ wells on plates containing 100 $\mathrm{mM}$ sodium acetate, $\mathrm{pH} 4.6,0.5 \%$ polygalacturonic acid, and $0.8 \%$ agarose. Plates were incubated for $12 \mathrm{~h}$ at $30^{\circ} \mathrm{C}$, and the halo caused by enzyme activity was visualized after $5 \mathrm{~min}$ of treatment with $6 \mathrm{~N} \mathrm{HCl}$. PG activity was expressed as agarose diffusion units, with 1 agarose diffusion unit defined as the amount of enzyme that produced a halo of $0.5-\mathrm{cm}$ radius (external to the inoculation well) after $12 \mathrm{~h}$ at $30^{\circ} \mathrm{C}$.

\section{Expression analysis.}

Three rosette leaves from three individual plants or about 15 seedlings for each treatment were collected, frozen in liquid nitrogen, and homogenized with mortar and pestle. Total RNA was extracted using $1 \mathrm{ml}$ of Tri-reagent (Sigma) per $100 \mathrm{mg}$ of tissue. RNA was separated on a formaldehyde-agarose gel, was transferred to nylon membrane, and was hybridized with radioactive probes, as previously described (Reuber and Ausubel 1996). Prior to hybridization, equal loading and transfer of RNA to membrane was verified by staining ribosomal RNA with an aqueous solution containing $0.03 \%$ methylene blue, $0.3 \%$ sodium acetate, $\mathrm{pH} 4.7$, followed by extensive washing with distilled water (Sambrook et al. 1989). A ${ }^{32}$ P-labeled antisense-specific AtPGIPl probe was prepared by asymmetric PCR (primer sequence, 5'-TTGAAATTTGCAAGTAAACC-3'), using as a template the $3^{\prime}$-untranslated region portion of the gene (Ferrari et al. 2003b). The probe was purified using ProbeQuantTM G50 micro-columns (Amersham Pharmacia Biotech, Uppsala, Sweden). Blots were washed once for $20 \mathrm{~min}$ at room temperature with $2 \times \mathrm{SSC}(1 \times \mathrm{SSC}$ is $0.15 \mathrm{M} \mathrm{NaCl}$ plus $0.015 \mathrm{M}$ sodium citrate), $0.1 \%$ sodium dodecyl sulfate (SDS) and twice for 20 min at $65^{\circ} \mathrm{C}$ with $0.1 \times \mathrm{SSC}, 0.1 \% \mathrm{SDS}$. Images were taken with a Typhoon 9200 Phosphorimager (Amersham Biosciences, Buckinghamshire, U.K.) after overnight exposure.

For quantitative RT-PCR analysis, RNA was treated with Turbo-DNase I (Ambion, Austin, TX, U.S.A.), and first-strand cDNA was synthesized, using ImProm-II reverse transcriptase (Promega, Madison, WI, U.S.A.) following the instructions of the manufacturer. Real-time PCR analysis was performed using an iCycler (Biorad) following the instructions of the manufac- 
turer. cDNA ( 2 to $3 \mu \mathrm{l}$, corresponding to $120 \mathrm{ng}$ of total RNA) were amplified in $30 \mu \mathrm{l}$ of reaction mix containing $1 \times$ IQ SYBR Green supermix (Biorad) and $0.4 \mathrm{mM}$ of each primer. The primers used were as follows: 5'-GACATTGTGTCTCTT GTTCTTGTTC-3' and 5'-CCGGAGAATATGGTTAAGGCG-3' for AtPGIP1; 5'-TGAACTTAGTAGGAACAAGC-3' and 5'GCCTAGTGACTTTGGTATAG-3' for AtPGIP2; 5'-GGAAG AAGAAGACTTACACC-3' and 5'-AGTCCACACTTACCAC AGTA -3' for $U B Q 5$. Expression levels of each PGIP gene, relative to $U B Q 5$, were determined using a modification of the Pfaffl method (Pfaffl 2001). Briefly, average cycle threshold $\left(\mathrm{aC}_{\mathrm{T}}\right)$ was calculated for each gene from three replicates, corrected for the efficiency of PCR (E, discussed below) as follows: $\mathrm{aC}_{\mathrm{T}} \times \log _{2}(1+\mathrm{E})$, to obtain $\mathrm{C}_{\mathrm{T}} . \Delta \mathrm{C}_{\mathrm{T}}$ between either AtPGIP1 or $A t P G I P 2$ and $U B Q 5$ was then calculated for each sample, and expression levels for each $P G I P$ transcript were calculated as $2^{-}$ $\triangle \mathrm{CT}$ and were expressed in arbitrary units. Relative expression of the RT-PCR products in treated samples, as compared with the expression in the corresponding untreated samples, was determined using the $\Delta \Delta \mathrm{C}_{\mathrm{t}}$ method (Livak and Schmittgen 2001).

Amplification efficiency for AtPGIP1 and AtPGIP2 with the primers indicated above was determined using serial dilutions of cDNA samples and was comparable; E was 0.97 to 0.98 for both genes, with a high linearity of amplification (Pearson correlation coefficient $r>0.95$ ). Amplification efficiency for the two genes was also tested using recombinant pBlueScript II SK plasmids containing the cloned cDNAs (Ferrari et al. 2003b) as templates and the gene for ampicillin resistance as a reference gene. Efficiency was similar for AtPGIP1 and AtPGIP2, allowing us to compare their transcript levels in each RNA sample.

\section{ACKNOWLEDGMENTS}

We would like to thank L. Tufano and D. Pontiggia for their skillful technical assistance. Research was supported by the Institute Pasteur-Fondazione Cenci-Bolognetti and the MInistero per l'Istruzione, l'Università e la Ricerca Scientifica (FIRB RBNE01KZE7_005).

\section{NOTE ADDED IN PROOF}

The Colletotrichum isolate here indicated as C. acutatum SHK788 has been reclassified as C. lupini var. setosum (Lotter, H. C., and Berger, D. K., 2005. Anthracnose of lupins in South Africa is caused by Colletotrichum lupini var. setosum. Australas. J. Plant Pathol. 34:385-392).

\section{LITERATURE CITED}

Alonso, J. M., Hirayama, T., Roman, G., Nourizadeh, S., and Ecker, J. R. 1999. EIN2, a bifunctional transducer of ethylene and stress responses in Arabidopsis. Science 284:2148-52.

Beffa, R. S., Neuhaus, J. M., and Meins, F., Jr. 1993. Physiological compensation in antisense transformants: Specific induction of an "ersatz" glucan endo-1,3-beta-glucosidase in plants infected with necrotizing viruses. Proc. Natl. Acad. Sci. U.S.A. 90:8792-8796.

Bradford, M. M. 1976. A rapid and sensitive method for quantification of microgram quantities of protein utilizing the principle of protein-dye binding. Anal. Biochem. 72:248-254.

Cervone, F., Hahn, M. G., De Lorenzo, G., Darvill, A., and Albersheim, P. 1989. Host-pathogen interactions XXXIII. A plant protein converts a fungal pathogenesis factor into an elicitor of plant defence responses. Plant Physiol. 90:542-48.

Cervone, F., De Lorenzo, G., Pressey, R., Darvill, A. G., and Albersheim, P. 1990. Can Phaseolus PGIP inhibit pectic enzymes from microbes and plants? Phytochemistry 29:447-9.

Clough, S. J., and Bent, A. F. 1998. Floral dip: A simplified method for Agrobacterium-mediated transformation of Arabidopsis thaliana. Plant J. 16:735-43.

D’Ovidio, R., Raiola, A., Capodicasa, C., Devoto, A., Pontiggia, D., Roberti, S., Galletti, R., Conti, E., O'Sullivan, D., and De Lorenzo, G. 2004. Characterization of the complex locus of bean encoding polygalacturonase-inhibiting proteins reveals subfunctionalization for defense against fungi and insects. Plant Physiol. 135:2424-2435.
De Lorenzo, G., R. Castoria, D. Bellincampi, and F. Cervone. 1997. Fungal invasion enzymes and their inhibition. Pages 61-83 in: The Mycota. G. Carroll and P. Tudzynski (eds.). Springer-Verlag, Berlin.

De Lorenzo, G., D’Ovidio, R., and Cervone, F. 2001. The role of polygalacturonase-inhibiting proteins (PGIPs) in defense against pathogenic fungi. Annu. Rev. Phytopathol. 39:313-335.

Denby, K. J., Kumar, P., and Kliebenstein, D. J. 2004. Identification of Botrytis cinerea susceptibility loci in Arabidopsis thaliana. Plant J. 38:473-486.

Epple, P., Apel, K., and Bohlmann, H. 1997. Overexpression of an endogenous thionin enhances resistance of Arabidopsis against Fusarium oxysporum. Plant Cell 9:509-20.

Federici, L., Di Matteo, A., Fernandez-Recio, J., Tsernoglou, D., and Cervone, F. 2006. Polygalacturonase inhibiting proteins: Players in plant innate immunity? Trends Plant Sci. 11:65-70.

Ferrari, S., Plotnikova, J. M., De Lorenzo, G., and Ausubel, F. M. 2003a. Arabidopsis local resistance to Botrytis cinerea involves salicylic acid and camalexin and requires EDS4 and PAD2, but not SID2, EDS5 or PAD4. Plant J. 35:193-205

Ferrari, S., Vairo, D., Ausubel, F. M., Cervone, F., and De Lorenzo, G. 2003 b. Tandemly duplicated Arabidopsis genes that encode polygalacturonaseinhibiting proteins are regulated coordinately by different signal transduction pathways in response to fungal infection. Plant Cell 15:93-106.

Huang, Q., and Allen, C. 2000. Polygalacturonase are required for rapid colonization and full virulence of Ralstonia solanacearum on tomato plants. Physiol. Mol. Plant Pathol. 57:77-83.

Isshiki, A., Akimitsu, K., Yamamoto, M., and Yamamoto, H. 2001. Endopolygalacturonase is essential for citrus black rot caused by Alternaria citri but not brown spot caused by Alternaria alternata. Mol. Plant Microbe Interact. 14:749-757.

Jang, S., Lee, B., Kim, C., Kim, S. J., Yim, J., Han, J. J., Lee, S., Kim, S. R., and An, G. 2003. The OsFOR1 gene encodes a polygalacturonaseinhibiting protein (PGIP) that regulates floral organ number in rice. Plant Mol. Biol. 53:357-369.

Kars, I., Krooshof, G. H., Wagemakers, L., Joosten, R., Benen, J. A., and van Kan, J. A. 2005. Necrotizing activity of five Botrytis cinerea endopolygalacturonases produced in Pichia pastoris. Plant J. 43:213-225.

Livak, K. J., and Schmittgen, T. D. 2001. Analysis of relative gene expression data using real-time quantitative PCR and the $2^{-\Delta \Delta C T}$ method. Methods 25:402-408.

Madlung, A., and Comai, L. 2004. The effect of stress on genome regulation and structure. Ann. Bot. (London) 94:481-495.

Manfredini, C., Sicilia, F., Ferrari, S., Pontiggia, D., Salvi, G., Caprari, C., Lorito, M., and De Lorenzo, G. 2005. Polygalacturonase-inhibiting protein 2 of Phaseolus vulgaris inhibits BcPG1, a polygalacturonase of $\mathrm{Bo}$ trytis cinerea important for pathogenicity, and protects transgenic plants from infection. Physiol. Mol. Plant Pathol., 67:108-115.

Meza, T. J., Kamfjord, D., Hakelien, A. M., Evans, I., Godager, L. H., Mandal, A., Jakobsen, K. S., and Aalen, R. B. 2001. The frequency of silencing in Arabidopsis thaliana varies highly between progeny of siblings and can be influenced by environmental factors. Transgenic Res. 10:53-67.

Oeser, B., Heidrich, P. M., Muller, U., Tudzynski, P., and Tenberge, K. B. 2002. Polygalacturonase is a pathogenicity factor in the Claviceps purpurea/rye interaction. Fungal. Genet. Biol. 36:176-186.

Pfaffl, M. W. 2001. A new mathematical model for relative quantification in real-time RT-PCR. Nucleic Acids Res. 29:e45.

Reuber, T. L., and Ausubel, F. M. 1996. Isolation of Arabidopsis genes that differentiate between resistance responses mediated by the RPS2 and RPM1 disease resistance genes. Plant Cell 8:241-9.

Sambrook, J., E. F. Fritsch, and T. Maniatis. 1989. Molecular Cloning: A Laboratory Manual. Cold Spring Harbor Laboratory Press, Cold Spring Harbor, NY, U.S.A.

Shieh, M. T., Brown, R. L., Whitehead, M. P., Cary, J. W., Cotty, P. J., Cleveland, T. E., and Dean, R. A. 1997. Molecular genetic evidence for the involvement of a specific polygalacturonase, P2c, in the invasion and spread of Aspergillus flavus in cotton bolls. Appl. Environ. Microbiol. 63:3548-3552.

Taylor, R. J., and Secor, G. A. 1988. An improved diffusion assay for quantifying the polygalacturonase content of Erwinia culture filtrates. Phytopathology 78:1101-1103.

ten Have, A., Mulder, W., Visser, J., and van Kan, J. A. L. 1998. The endopolygalacturonase gene Bcpgl is required for full virulence of Botrytis cinerea. Mol. Plant Microbe Interact. 11:1009-16.

Terras, F. R., Eggermont, K., Kovaleva, V., Raikhel, N. V., Osborn, R. W., Kester, A., Rees, S. B., Torrekens, S., Van Leuven, F., Vanderleyden, J., Cammue, B. P. A., and Broekaert, W. F. 1995. Small cysteine-rich antifungal proteins from radish: Their role in host defense. Plant Cell 7:573-588.

Zhu, B., Chen, T. H., and Li, P. H. 1996. Analysis of late-blight disease resistance and freezing tolerance in transgenic potato plants expressing sense and antisense genes for an osmotin-like protein. Planta 198:70-77. 